\title{
A 3D Simulation System for Wireless Communication
}

\author{
Jingbo Mao \\ The Second Artillery College, China
}

\author{
Lin Wang \\ Wuhan Communication Academy,China
}

\author{
Yan Meng \\ The Second Artillery College,China
}

\begin{abstract}
The paper discussed the key techniques to build 3D simulation environment for wireless communication system based on Vega Prime and HLA. The LOD with 2D symbols and size-keeping rendering for communication entities are designed to improve the usability and intelligibility. The invisible electromagnetic wave beams are categorized by their vivid shapes and constructed by the extension of VSG, then the elaborate line-step-alpha and sine-alpha strip textures are built and rendered dynamically to represent the flowing wave. These flexible and applicable strategies are applied to integrate the complex user space environment associated with the land, sea, air, space and electronic scenarios.
\end{abstract}

Keywords-3D simulation, Wireless Communication, Wireless Wave Beam, Vega Prime

\section{INTRODUCTION}

Wireless communication is widely used in military, so in war-game simulation the capability of the information exchange becomes the necessary attribute for the simulation entities. With the development of the scene simulation technology and the distributed interactive simulation technology, we have build an advanced practicable tactical scenario generating systems based on GIS/HLA, integrated the complex user space environment associated with the land, sea, air, space and electronic scenarios. Many kinds of motional and static entities are modeled and simulated. During the simulation advancing, these entities need to exchange information with each other to complete their tasks by the wireless communication system, so the wireless transmitters and receivers are modeled too. And based on the actual GIS information, the communication links are computed in real-time to evaluate the performance of the separate links or networks (or sub-networks). Currently, we are building a 3D simulation system/federate for the simulation federation based on Vega Prime. The 3D federate reflects the information from the HLA interface, and dynamically renders the corresponding entities in the virtual scene. But, as we know, the electromagnetic wave or the wireless links between transmitters and receivers cannot be seen by our eyes, so it is most difficult to understand them, descript them, and draw them. The modeling and simulation for the invisible beam wave are discussed mainly in this paper.

\section{SYSTEM DESIGN}

The 3D simulation system for wireless communication system is designed according to standardized software engineering, and includes four components:

- Main application component, the main frame of the 3D simulation system based on MFC and Vega Prime,

- Large scale terrain manager component, a dynamic library packing the Vega Prime LADBM (Large Area Database Management),

- Communication simulation component, a dynamic library extended from VSG(Vega Scene Graph).

- HLA Interface component, a dynamic library for the MOM (Management Object Model) classes, the customized object classes and interaction classes.

\section{DESIGN AND IMPLEMENTATION OF THE KEY} TECHNOLOGIES

A. Building and Loading of Large Area Terrain

Vega Prime Large Area Database Management (LADBM) is specifically designed to facilitate the development and deployment of applications that include very large and complex databases. Correspondingly, the Creator Terrain Studio (CTS) is a powerful tool which offers users a hierarchical, top-down workflow to generate terrain database automatically. So the most arduous work is to collect the satellite photos and DEM (Digital Elevation Model), and then build the large area terrain by CTS. We have built the natural environment with multi-resolution and multi-area data source, so the rendering performance is improved dramatically.

\section{B. Modeling and Rendering for Simulation Entities}

Simulation entities, such as ground vehicles, ships, aircrafts, are modeled in Creator, then exported in OpenFlight format. Like general LOD(Level of Detail), these 3D models are modeled in multi-resolution to improve the performance. However, when too many entities, especially the same category entities, are displaying simultaneously in the large area terrain, and the eye point is so far, as a result, these entities are too small to distinguish. But on the other hand, we desire to know where the simulation entities are, so we add the text names of the entities upper side of the 3D model. These text labels are designed as vsBillboard nodes created dynamically by the application, added to the corresponding entity vpObject as 
the least detail switch node, and positioned according to its parent node. Furthermore, these billboards should keep a certain fixed size which is computing in real-time according to the distance factor. To facilitate the computation, the size and the distance are in direct ratio and the ratio factor can be adjusted by user.

\section{Modeling and Simulation for Communication \\ Equipments}

As a build-in child object of the general simulation entities, the communication equipments, such as sensors, radars, wireless transmitters and receivers, can emit or receive electromagnetic wave, so the separate wireless links, even the wireless networks can be created. The simulation entities communicate with each other through the invisible links and determined their moving route by the performance of the links. For example, in mountain areas, if they are interrupted frequently because of the terrain, we should try another route or supplement another land or air stations which receive wireless signals from one vehicle and retransmit to the other one.

As for the hardware, the wireless communication entities are organized into four main categories: wireless transmitters, wireless receivers, sensors, radars. Some subcategories are considered. For instance, the transmitters included satellite transmitter or retransmitter, broadcasting station transmitter, infrared transmitter, interfering transmitter, and so on.

From the communication or mathematic point of view, the communication-related entities are modeled based on classic wireless communication theory. For each entity, the signal source encoding mode(PCM, $\triangle \mathrm{M}$, etc.), modulation mode(BPSK, QPSK, FSK, etc.), filter mode(Gaussian filter, Butterworth filter, Bessel filter, etc.), channel coding mode(linear block code, convolution code, etc.), antenna mode(circular antenna, rectangular antenna, isotropic antenna, parabolic antenna, etc.), and some manual gains and losses are considered.

From the software engineering point of view, these communication equipments are designed and implemented as $\mathrm{C}++$ objects, and can be reused as child objects of other type simulation entities, such as fixed VSAT earth station.

In the simulation cycle, their motional statuses are determined by their parent node. Combining the communication theory formulas and GIS terrain access computing, the performance of the communication links, such as $\mathrm{C} / \mathrm{N}, \mathrm{Eb} / \mathrm{N} 0$, can be computed in real-time, and reported in graphics, text, or grid report.

D. Modeling and Simulation for Wireless Wave Beam

1) Shape of Wireless Wave Beam

The wireless wave beam emitted from the transmitter is different from various antennas. The isotropic antenna has sphere beam. The directional antenna has a specific beam for their different antenna gain contours. Different beam shape (e.g. cylinder, cone, pyramid) represents different electromagnetic wave emitted from user defined wireless communication equipment. Generally, the beam shape of the directional antenna are complex and difficult to understand, so in order to render them expediently and vividly in virtual scene, five beam shapes or volumes modes are modelled:

- Simple cylinder beam

Simple cylinder beam is defined by Radius, Min Range and Max Range. Radius determines its thickness, and the Height, difference between Max Range and Min Range is the effective display range.

- Simple circular cone beam

Simple circular cone beam is the simplest standard circular cone shape, the Cone Angle defines the beam width, the Min Range defines the start point and the Max Range defines the stop point. The Height, difference between Max Range and Min Range is the effective display range. For flexibility, Start and Stop Angle are defined to make the cone not orbicular. By default, Start Angle is 0 degree, and Stop Angle is 360 degree.

- Complex circular cone beam

Complex circular cone beam is similar to simple circular cone beam, but it has tow cone boundaries: an inner one and an outer one. The effective area is between the cones,as shown in Figure.1.

- Simple pyramid beam

Simple pyramid beam is the simplest standard rectangular or pyramid shape, the Horizontal Angle and Vertical Angle parameters are used to define the rectangle,as shown in Figure.2.

- Complex pyramid beam

Complex pyramid beam is similar to the simple pyramid beam. It has an inner pyramid which define by the inner horizontal angle and inner vertical angle, and an outer pyramid which is defined by the outer horizontal angle and outer vertical angle, so it is displayed as a rectangular hole inside.

2) Visualization of Wireless Wave Beam

As a leading product in real-time 3D simulation solutions, Vega Prime is the most flexible, extensible commercial-off-the-shelf (COTS) tool available for the creation and deployment of visual simulation. But it still lacks special effects to render the wireless links or sensor beam. Fortunately, Vega Prime has an extendable architecture, and we can customize our application by VSG to build the wireless wave beam dynamically. [1][2]

(1)Building the Geometries

Generally, main body surface can represent the beam effect. But, In fact, we build three geometries to represent one beam: main body geometry, bottom boundary geometry and side line geometry.

Main body geometry is the major visible part. Obviously, the surface is not convergent and can be subdivided into many quadrilaterals. So the major task is to build the vertexes of the quadrilaterals (vrGeometry::PRIMITIVE_QUAD). When computing the coordinates of the vertexes, the bottom boundary geometry and side line geometry are created as a by-product. Bottom boundary geometry is the line strip (vrGeometry::PRIMITIVE LINE STRIP), and the side line geometry is the discrete lines (vrGeometry::PRIMITIVE_LINE). [1][2] 
As expedited above, the communication equipments are designed to be a child, so its corresponding beam geometry should be a child of the equipment or the platform 3D model. On the other hand, the beam can be transformed in its body coordinates. So we inherit the beam classes from vsTransform class.vsBaseBeam is the base class for our beam node, it encapsulates the common properties for all kinds of beams, such as min range, max range, scan mode, pointing mode, texture rendering mode. Each type beam inherits from it and the major task of the subclasses is to build the main body surface quadrilaterals, the bottom line strip, and the side lines. Theoretically, any shape can be created. If the geometry can be expressed by some math equation, it is easier to compute the vertexes. Programmers only need to adjust the parameters to make the greatest performance and the greatest effect.

(2) Building the colored stripped textures

The textures of the geometries are dynamically created, and users can set its colour and transparency mode. The color is expressed in RGB mode. In fact, the texture is homochromous. So the transparency mode is used to enhance the effect, and include two modes:

Step line mode: it has only two transparency alpha values: 0 and 1 , consequently the texture is rendered like a stripe, just as STK Sensor box pulse.

Sine gradual mode: as its name suggested, the transparency alpha value are gradual varied by sine function. It makes the main body surface transition smoothly.

(3)Animating the textures drawing

When the electromagnetic wave is propagating, the beam strip is flowing smoothly along the wave direction. In $3 \mathrm{D}$ computer graphics, it is equivalent to the dynamic texture. So the beam rendering strategy is defined to make the texture move with a specified speed in the update frame.

The vsWaveAnimation class is inherited from vrRenderStrategy, and overrides the draw method where the current texture matrix is modified and applied to
vrDrawContext. So the new texture coordinates are active and the texture will flow. [1][2]

\section{HLA INTERFACE COMPONENT}

HLA interface component provides the 3D simulation system ability to interchange information with other federates by HLA RTI. The object classes in 3D simulation system are different from those in the scenario generating system. To improve the system reusability and composability, the component is separated from the 3D simulation system. Currently, the component is suite for our private scenario editor. For other simulations, other FOMs, the modification is needed. However, we provide many common classes (e.g. dynamic object class, static object class) to make the least modification.[3]

\section{CONCLUSION}

This paper discusses the $3 \mathrm{D}$ visualization techniques for wireless communication entities and invisible electromagnetic wave. For communication entities, the special LOD and size-keeping strategies are applied to improve the usability and intelligibility. For invisible electromagnetic wave, some beam patterns are designed and corresponding geometries are modelled, at the same time the step line and sine style alpha-stripped textures are built and mapped. The textures matrix is modified in update frame to animate the wave strips dynamically. These strategies are flexible and applicable for the integration of the complex user space environment associated with the land, sea, air, space and electronic scenarios.

\section{REFERENCES}

[1] MultiGen-Paradigm Inc., Vega Prime Programmer's Guide, Version $2.0[\mathrm{M}], 2005$.

[2] MultiGen-Paradigm Inc., VSG ${ }^{\mathrm{TM}}$ (Vega Scene Graph $^{\mathrm{TM}}$ ) version 2.0: Interworkings and Extensibility Mechanisms, Version 2.0[M], 2005.

[3] Pitch AB, Users Guide to pRTI ${ }^{\mathrm{TM}}$ 1.3, 2007.

[4] DMSO, High Level Architecture Run-Time Infrastructure Programmer's Guide [DB/OL], 21 September 1998.

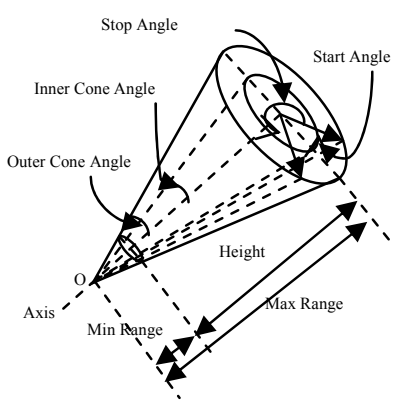

Figure 1. Complex Circular Cone Beam

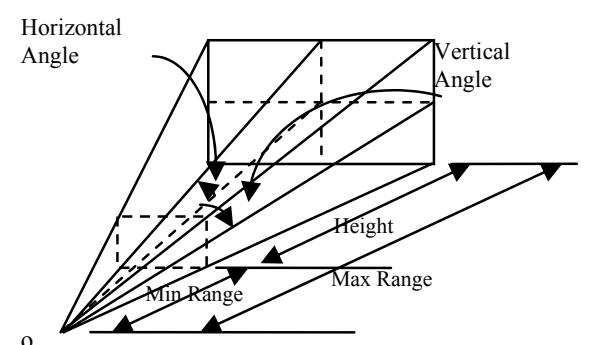

Figure 2. Simple Pyramid Beam 